\title{
Preliminary Behavior of Chinkara (Gazella Bennettif) under Captive Conditions with Future Conservation Strategies
}

Muhammad Idnan ${ }^{1,3, *}$, Arshad Javid, Muhammad Nadeem4, Ali Hussain', Sajid Mansoor², Waqas Ali', Syed Mohsin Bukhari ${ }^{1}$

1Department of Wildlife and Ecology, University of Veterinary \& Animal Sciences, Lahore, Pakistan 2Department of Microbiology, Faculty of Life science, University of Central Punjab, Lahore, Pakistan

3Department of Zoology, Faculty of Sciences, University of Central Punjab, Lahore, Pakistan

${ }^{4}$ Department of Dairy Technology, University of Veterinary \& Animal Sciences, Lahore, Pakistan

\section{ABSTRACT}

Background: Study of behavioral conformations play a significant role in ex-situ conservation of ungulates particularly of deer to propagate deer farming. Due to advancement in animal hunting techniques, captive breeding is the best solution to propagate animals for sports or other traditional \& medicinal uses. Due to the lack of management methods, captive breeding programs have been used on a trial and error basis for the rearing of wild animals like Chinkara.

Objectives: The present study was planned to investigate behavioral patterns in captivity to explore factors over the reproductive success of the Chinkara and the potential of this wild animal (Chinkara) for deer farming practices in Pakistan.

Methodology: Present study was conducted on the behavior of Chinkara (Gazella bennettii) [wild-caught $(W C)=20$, captive-bred $(C B)=10$ ] for a period of one year from April 2013 to March 2014 at captive breeding facilities for ungulates, Ravi campus Pattoki, the University of Veterinary and Animal Sciences (UVAS), Pakistan.

Results: The behavior of captive-born and wild-caught animals was observed by focal sampling pattern to frame conservation strategies for successful management practices for the promotion of deer farming in Pakistan. Similar behavioral patterns were observed in both WC and CB animals but WC male Chinkara displayed a higher degree of agonistic interaction than CB males. From these results, it is predicted that there is no obvious immediate effect of captivity on behavioral configurations up to 10 generations in Chinkara.

Conclusion: It is suggested that Chinkara is not suitable for domestication like Goat until further studies on the Ethology of Chinkara.

Keywords

Chinkara (Gazella bennettii), Ex Situ

Conservation, Deer Farming, Wildlife

Management, Captivity, Domestication.

Cite this article: Idnan M, Javid A, Nadeem M, Hussain A, Mansoor S, Ali W, Bukhari SM. Preliminary Behavior of Chinkara (Gazella Bennettii) under Captive Conditions with Future Conservation Strategies. RADS J Biol Res Appl Sci. 2020; 11(1):19-26.

This is an Open Access article distributed under the terms of the Creative Commons Attribution License (http://creativecommons.org/licenses/by/4.0), which permits unrestricted use, distribution, and reproduction in any medium provided the original work is properly cited.

\section{INTRODUCTION}

The Chinkara (Gazella bennettii) is one of the ungulates belonging to the genus Gazelle which is found in different
Article info.

Received: October 29, 2018

Accepted: July 26, 2020

\section{Funding Source: Nil \\ Conflict of Interest: Nil}

habitats like flat plains, grasslands, sand deserts, hilly areas, dry scrubs and light forest of South Asia, India, 
parts of Iran and Pakistan ${ }^{1}$. This animal is distributed much around central and western India, spreading through Pakistan, south-western Afghanistan into northcentral Iran. In the Thar desert of India, about 1000,000 Chinkara has been estimated ${ }^{2}$. While a much less number is estimated in Iran 2,818. The current population status of Chinkara in Afghanistan is unknown but they are considered to be scarce 3 . Due to overhunting in Pakistan, the numbers of Chinkara are declining with no exact estimate 4 .

Massive hunting has severely embellished Gazelle's population in Afghanistan, Iran and Pakistan, where they are hunted for meat and trophy. Chinkara is a facultative drinker and can survive in very arid areas. In deserts, they occasionally graze cultivated fields with rape seeds and sorghum ${ }^{3}$. The animal is susceptible to feral dogs, habitat destruction and poaching for meat consumption ${ }^{5}$.

Management of endangered species in captivity is of vital importance for a sustainable wildlife management practice; nonetheless, an increase in captive production can be a challenging task for wildlife managers due to insufficient information regarding the breeding behavior, biology of the species concerned and the difficulty of estimating different management patterns. Even with vulnerability and complexity, decision-analytic approaches can be used to recognize optimum management decisions to increase the captive production ${ }^{6}$.

In Asia, the majority of the herbivores is under threat due to hunting, poaching, grazing competition with livestock and habitat fragmentation etc. Conservation of Asiatic ungulates is frequently apprehensive by poor information regarding the population dynamics and delicate evaluation of specific threats. Wildlife conservation programs involve species-specific information and understanding of all concerned complications that may reduce population growth 7 . The present study is therefore planned to investigate behavioral patterns in captivity to explore those factors over the reproductive success of the Chinkara and the potential of this wild animal (Chinkara) for deer farming practices in Pakistan.

\section{MATERIALS AND METHODS}

\section{Study Area}

The study was conducted at captive breeding facilities for ungulates at the University of Veterinary and Animal Sciences (UVAS), Lahore, Ravi Campus, district Kasur, Pakistan. The development of the irrigation system like the canal's system has resulted in the development of vast agricultural areas for farming and gardens or flower's nurseries of the city at the expense of the Sub Tropical Thorn Forest eco-zone. Agricultural farmlands, flower farms and canals in adjoining areas constitute a subtropical thorn forest biome in the study area.

\section{Animals Studied}

A total of 30 animals were observed in this study. Animals were housed in two separate enclosures $A$ and $B$ with an equal ratio of males and females. The animals were captured from a semi-wild habitat and housed in enclosures $(100 \mathrm{ft} . \times 200 \mathrm{ft})$ with a well-ventilated shelter $(20 \mathrm{ft} . \times 20 \mathrm{ft})$. The shelter was constructed for animals to seek a save place in harsh weather condition. Enclosures were also having brick's wall of four feet in height with wire netting separated the enclosures. It assisted the animals to smell, hear and sight each other. One year before the start of the study Chinkara were housed in these enclosures. During the study period, eight Chinkara gave birth to young ones in a healthy condition. The animals were observed by similar people on each day and animals were identified with visual cues or identification marks by the observer. Randomly a focal animal was selected from the group to observe various behavioral frequencies and different behavioral parameters as mentioned in Table 1, were recorded continuously for $5 \mathrm{~min}$. A researcher started to observe the animals after $20 \mathrm{~min}$ of his entrance into the enclosure as the animals at their first notice of an invader started to alert others by a tail wagging, producing a sound of cheenk-cheenkcheenk, which is the reason that the animals are named as Chinkara and erecting their ears about the arrival of an observer. After a period of $20-30 \mathrm{~min}$, animals started to take fodder and performed other activities normally without showing any obvious notice of the intruders. The behavioral sampling did not affect the normal activities of the animals. Different behavioral parameters observed by 
a focal person 05 times in a day, 6 days in a week and for 12 weeks in a period of one year.

\section{Opportunistic Focal Animal Sampling}

All opportunistic recordings were considered independent observations. Chinkara lives in a group of 3 or 5 or sometimes solitary ${ }^{9}$ and the same behavior of grouping were observed in captivity. Random encounters of Chinkara were sought, followed by efforts to get the animal habituated to the observer's presence. Once sited, individuals were seen as long as the observer was tolerated by the animal. Those animals that ran away immediately after being noticed were excluded from the analysis as it was difficult to judge their activity in the moments before they escaped. The animals have not separated aside from required therapeutic care or planned accomplishments for management purpose.

\section{Feeding and Enclosure Management}

The animals were provided with fresh fodder, leaves of trees and grasses during the study period. As the leaves of Melia azedarach (Dhreik) and Zizyphus jujube (Beri) were abundant and were easily collected from the university area so that they were provided in addition to other forage. On daily basis, fresh and clean water was provided to the animals' ad libitum.

\section{Statistical Software}

SPSS 22 versions were used to analyze the data.

\section{RESULTS}

A comparison of the wild-caught (WC) and captive-bred (CB) Chinkara was observed. The WC males show significantly more agonistic behavior $(0.280 \pm 0.019)$ as compared to the individuals, who were born in captivity (CB) males $(0.213 \pm 0.071)(P<0.05)$. Usually, the $W C$ males displayed more agonistic interaction, anogenital sniffing, environmental sniffing, self-directed behavior and affinitive interaction than the $C B$ Chinkara. On the other hand, the $\mathrm{CB}$ animals tended to have a higher frequency of standing-alert and feeding/drinking activities than to WC Chinkara as mentioned in (Fig. 1) however, there is no statistically significant difference $(P>0.05)$ observed in behavioral parameters of Chinkara.

Table 1. The Average Values of Behavioral Parameters for Wild-Caught (WC-No.=20) and the Captive-Born (CBNo.= 10) Chinkara.

\begin{tabular}{ccccc}
\hline S. No. & Behavioral Parameters & Wild Born (WB) & Captive Born (CB) & P-value \\
\hline 01 & Resting Behavior (RE) & $0.391 \pm 0.031$ & $0.375 \pm 0.168$ & 0.873 \\
\hline 02 & Standing Alert Condition (SA) & $2.727 \pm 1.835$ & $2.919 \pm 1.267$ & 0.156 \\
\hline 03 & Locomotive Behavior (LO) & $4.436 \pm 1.401$ & $4.405 \pm 2.906$ & 0.913 \\
\hline 04 & Feeding/Drinking Behavior (FD) & $3.608 \pm 1.690$ & $4.250 \pm 1.731$ & 0.021 \\
\hline 05 & Rumination Activities (RU) & $0.463 \pm 0.136$ & $0.114 \pm 0.473$ & 0.022 \\
\hline 06 & Tail Pasting Behavior (TP) & ---------- & --------- & ---- \\
\hline 07 & Urinating/Defecation Process (UD) & $0.136 \pm 0.034$ & $0.166 \pm 0.087$ & 0.283 \\
\hline 08 & Environmental Sniffing Activities (ES) & $1.219 \pm 0.253$ & $1.057 \pm 0.495$ & 0.111 \\
\hline 09 & Self-directed Behavior (SD) & $1.138 \pm 0.430$ & $1.036 \pm 0.522$ & 0.351 \\
\hline 10 & Anogenital Sniffing Activities (AS) & $0.363 \pm 0.132$ & $0.294 \pm 0.105$ & 0.280 \\
\hline 11 & Affinitive Interaction (Al) & $0.202 \pm 0.135$ & $0.147 \pm 0.092$ & 0.153 \\
\hline 12 & Agonistic Interaction (Cl) & $0.280 \pm 0.019$ & $0.213 \pm 0.143$ & 0.071 \\
\hline 13 & Miscellaneous Behavior (MB) & $5.657 \pm 2.155$ & $5.527 \pm 1.206$ & 0.683 \\
\hline
\end{tabular}


The current study also prevails a difference in rumination behavior of WC Chinkara as compared to the captive breed (Fig. 2). The ruminants seek a safe place from predators and easily chew their cud in wild. That is why we have observed a high degree of rumination in Wildcaught animals as compared to the captive breed individuals. Contrary to this behavior, captive-born chinkara spent more time in foraging activities as compared to the wild-caught animals as depicted from Fig. 3, this might be due to non-exposure to their predators. Such a significant change in behavior plasticity provides the patterns for behavioral modifications to tame such wild animals and formulate strategies for deer farming, and subsequently ex-situ conservation for multipurpose aims, which may be discussed in other industrybased research strategies.

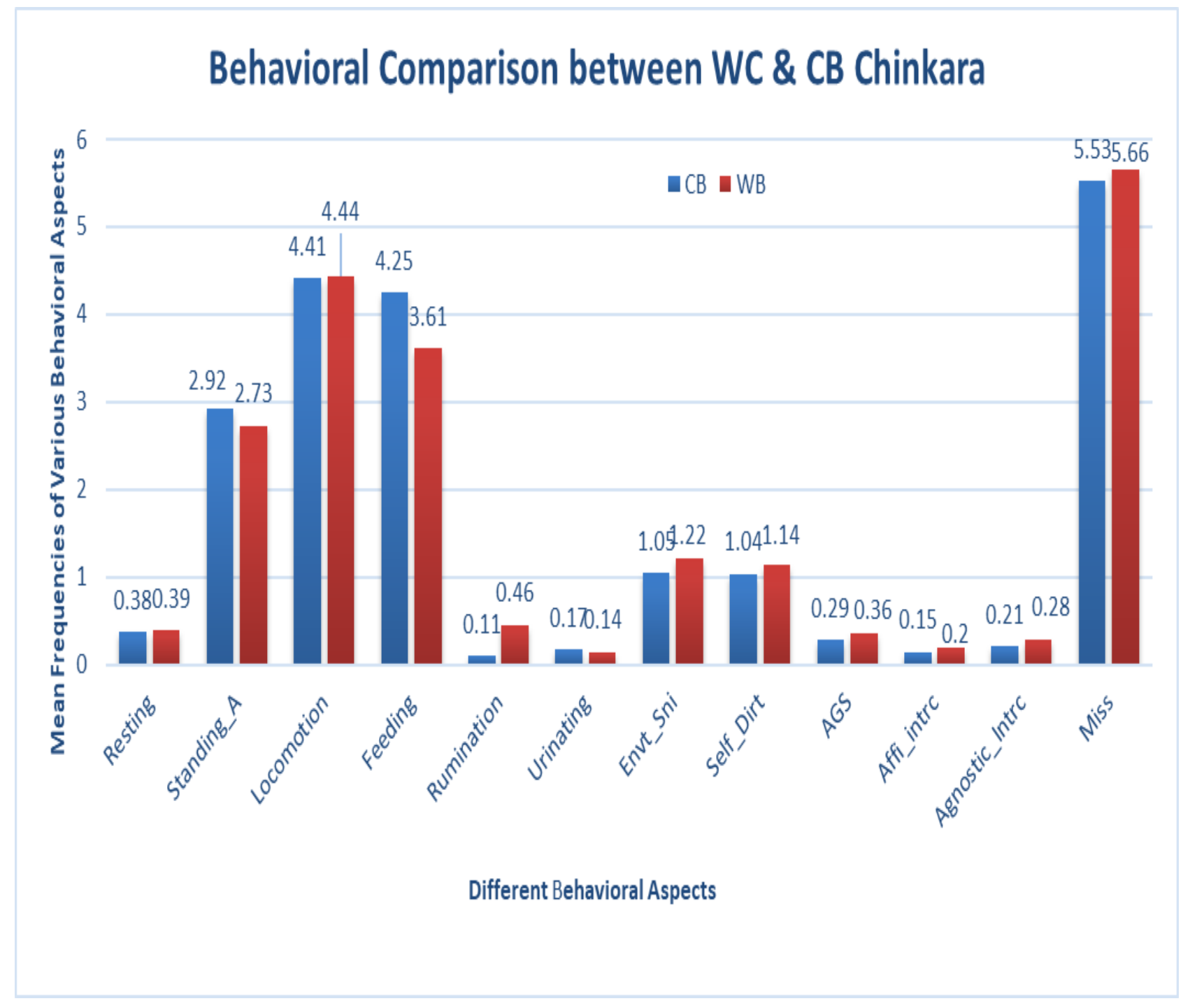

Figure 1. Behavioral parameters frequencies between captive-bred $(C B=10)$ and wild-caught $(W C=20)$ Chinkara. Behavioral parameters include Standing-alert (SA), Feeding \& Drinking (FD), Locomotion (LO), Urinating/Defecating (UD), Self -directed behavior (SD), Resting (RE), Environmental sniffing (ES), Ruminating (RU), Affinitive interaction (Al), Agonistic interaction (Cl), Ano-genital sniffing (AS) and Tail-pasting (TP). AS and TP were not included in the figure due to their increased frequency.

CB= Captive Born WB =Wild Born WC=Wild caught (Meng, et al. 2010) 


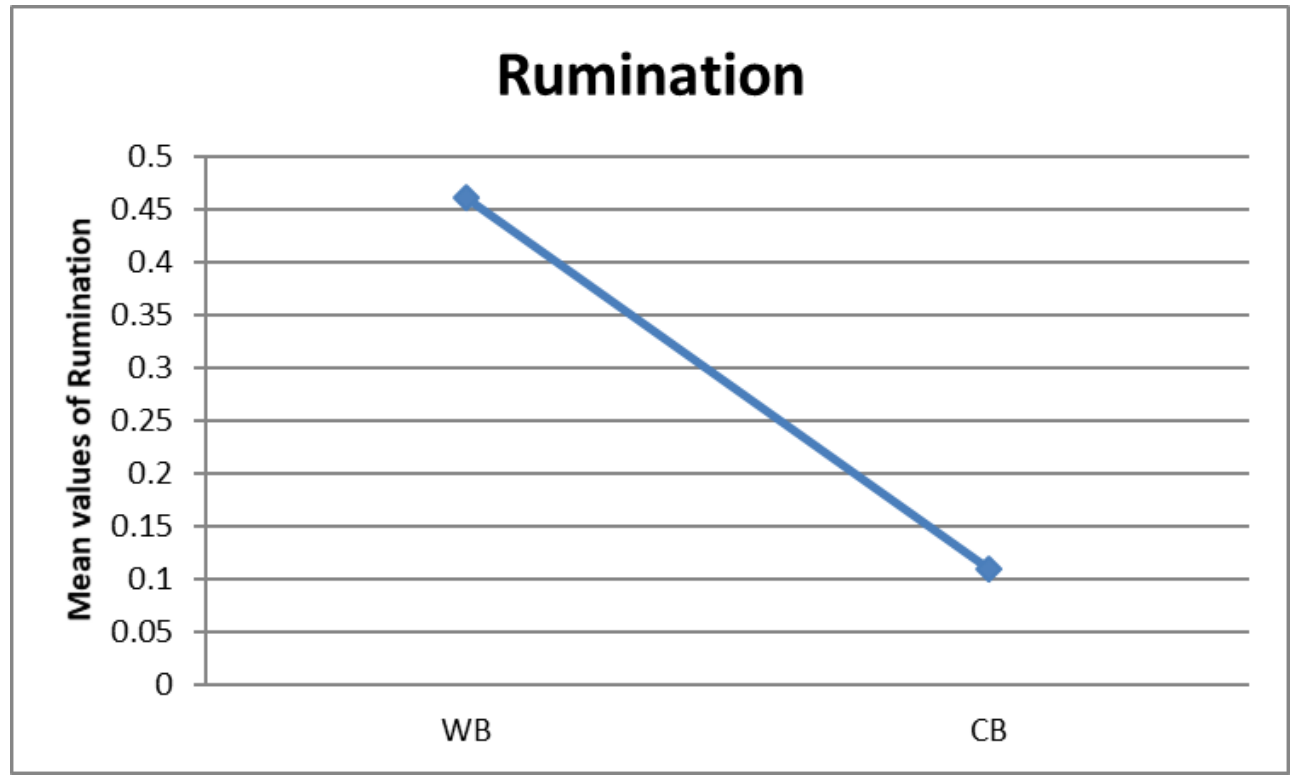

Figure 2. A comparison of rumination in wild-caught (WC) \& captive-breed (CB) Chinkara.

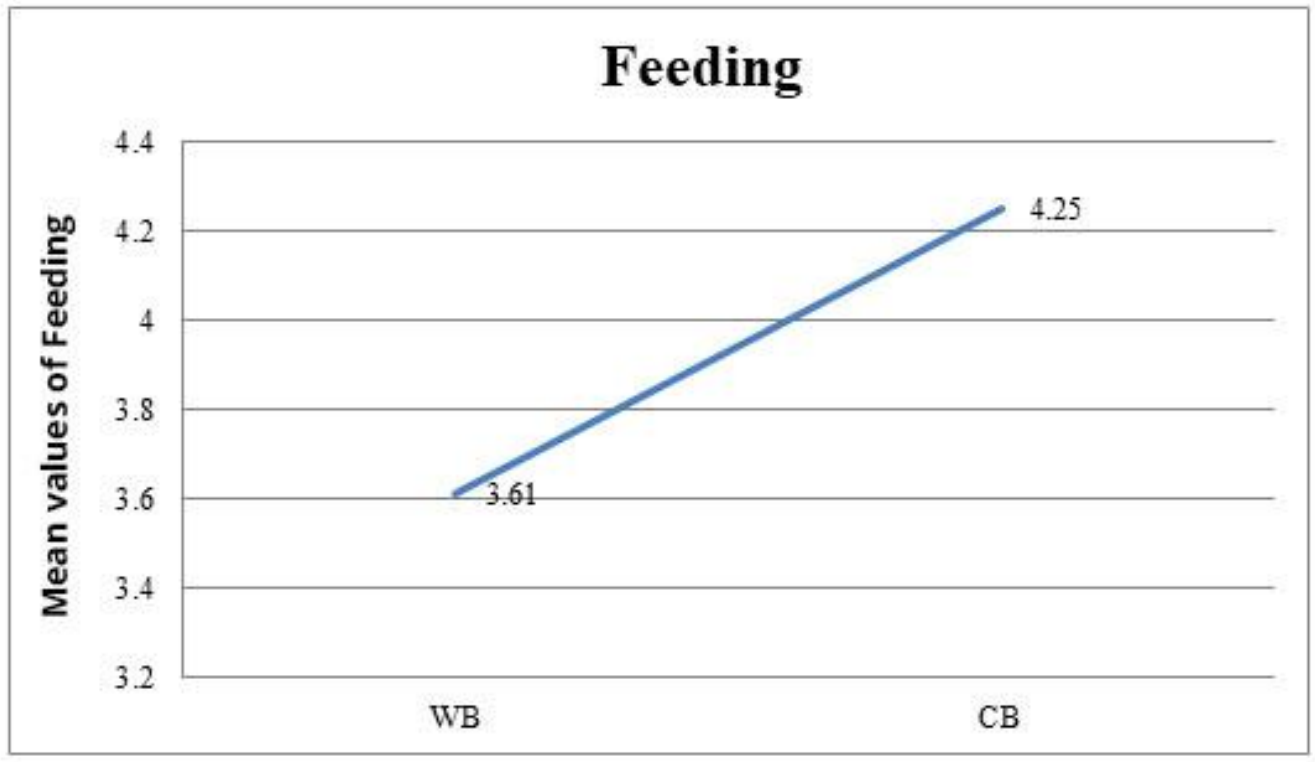

Figure 3. A comparison of feeding in wild-caught (WC) \& captive-breed (CB) Chinkara.

\section{DISCUSSION}

The behavioral modifications and genetic adaptations may be promoted by captive management through adaptations based on captive phenotypes ${ }^{10}$. Transfer of wild animals to captive conditions may result in differential selection pressures, change in environmental parameters such as availability of resources (water, food and mating partners) and proximity to perceived dangers is maximized by human exposure. In domestic animals, few behavioral traits are lost as compared to the observation of new behavioral traits. The behavioral differences are quantitative in wild and domestic stocks and could be described by threshold response or behavioral frequency ${ }^{11}$.

In the present study, no significant differences were observed for various behavioral categories in wild and captive-born Chinkara. Rumination in Wild-Born animals 
was more as compared to the captive breed individuals (Figure 2). This may be due to the reason from an evolutionary perspective that in a wild environment the ruminants find some safe place to hide from predators and chew their cud too. In captive conditions WC and CB born Chinkara were housed in identical enclosures, where they have no control over the number of co-inhabitants, but it was observed that the dominant male used to defend the female for mating with other males. This agonistic behavior has caused serious fights that resulted in deaths. It has been observed that the dominant male hits other males with horns at the abdominal and thoracic regions. It is noted that the social environment has a key role in the interaction of animals ${ }^{12}$. Results of preliminary agonistic behavioral interactions indicated that an unrestricted environment is not necessary by the factor for captive management.

Under captive conditions both the Wild-Born and CaptiveBorn Chinkara, which are confined to a similar condition in the study area, lacking ecological enrichment, are expected to involve more in agonistic interactions. This hypothesis is supported by current findings which showed the WB deer are considerably more aggressive than the CB Chinkara. Fear related behavior in wild animals has a genetic background and it plays an important role in the survival of animals in wild conditions and evolution as well12.

Besides, close interaction with people can be relied upon to cause an alteration of behavior, particularly distressing, where "selection pressure" may change i.e. aversion from people, who encounter the animals on daily basis for feeding and management related issues ${ }^{13}$. Wallace recommended that, after some generations, the absence of regular "selective pressure" may modify the genetic make-up of vital ethological characteristics ${ }^{14}$. In the present investigation, the focal person and guardians had to interact with Chinkara on daily basis for feeding and managing the enclosures of the animals. Such observations are encouraged by Zhang, who gave preparatory reports with regards to the domestication of musk deer ${ }^{15}$.

In our findings of various behavioral patterns in Chinkara, there were no apparent variations in general behavioral parameters like feeding, drinking, chasing, sniffing etc. but the differences in the social behavior i.e., affinitive $(0.202$ $\pm 0.135)$ and agonistic $(0.280 \pm 0.019)$ behavior in WB Chinkara were more than those observed in the CB Chinkara. On the contrary, it is shown in Figure 3 that captive breed individual spent more time on feeding which maybe because they are facing no predator exposure to predators under captive conditions while in wild conditions the organisms have to face such threats. In this study, no age effect was observed due to the small sample size. Taming and domestication of animals have been developed over a period of thousands of generations by human interaction and habituation with these animals for their commercial use. However, it is a difficult task to determine the degree of domestication of an animal because the phenotype of the animal not only depends on its genetics but also on the environment in which it is reared. Like in the study reported on foxes, it was observed that the foxes which were selected for domestication started to take food from caretakers and used to eat in human presence ${ }^{16}$. Nonetheless, it can be suggested from the results of our preliminary findings on behavioral modes for Chinkara in captivity that it has not been adaptive for behavioral modifications even for 10 generations born in captive conditions without proper taming practices. So, these results indicated that Chinkara is not suitable for domestication. But it has been observed that if the calves of Chinkara are kept from day one with other livestock and human beings they are tamed well and show no fear of human presence. Moreover, the animals reared under captive conditions do not learn how to survive in wild conditions, and by the presence of a significant predator, they may also face extinction. To protect such a vulnerable species other conservation measures should be taken into consideration so that the economics of deer farming would not be a question ${ }^{17}$. To ensure the long-term conservation of endangered animals a healthy stock of captive breed population should be maintained for release of captive animals in wild environment ${ }^{12}$.

In most social mammals, some females disperse from their natal group while others remain and breed there throughout their lives. In a few cases, females typically disperse after adolescence but the rest of them remain and breed in their natal group. This behavior is also observed in Chinkara deer under captive conditions which lessens the time to find a new mate for breeding. These 
contrasts in philopatry and dispersal have an important consequence on the kinship structure of groups which in turn, affects forms of social relationships between females ${ }^{18}$.

Conservation behavior is a relatively new interdisciplinary field that aims to investigate how proximate and ultimate aspects of animal behavior are formulated to prevent biodiversity loss. The usefulness of this new discipline is to promote practical conservation-matters, which is a topic to debate with some scientists arguing that the importance of behavior in conservation practice is exaggerated ${ }^{19}$.

Commonly it is observed that the captive-born animals are having deficiencies in foraging activities and locomotion as compared to the wild-born animals. Few of these discrepancies are continued in reintroduced animals for up to two years in the wild. Results of this research, however, provided little signs of farming or domestication and minor changes in behavior of captive and wild-born animals. These results showed the possibilities of reintroduction programs for conservation approaches, especially for the accomplishment of deer farming.

\section{CONCLUSION}

Domestication of Chinkara is a difficult task as various factors are involved in their behavioral modifications; the elements affecting the domestication process may be biological or ecological, enclosure size or management system and the development of a complicated relationship with human beings as well. From the results of this preliminary study, it is difficult to propose that chinkara is a suitable species for domestication. Further studies are recommended to find out stress level in wild chinkara to manipulate suitable managing practices.

\section{ACKNOWLEDGMENT}

The authors are thankful to all contributors and others from where the literature was cited.

\section{LIST OF ABBREVIATIONS}

Al Affinitive Interaction

AS Ano-genital Sniffing
CB Captive-Born

ES Environmental Sniffing

FD $\quad$ Feeding \& Drinking

LO Locomotion

SA Standing Alert

SD Self-Directed behavior

TP Tail-Pasting

UD Urinating-Defecating

WB Wild Born

WC Wild-Caught

\section{REFERENCES}

1. Khampariya PK, Singh SP. Status and feeding ecology of the Chinkara in Panna Forest, Panna (MP). Life Sci. Bul. 2011; 8:251-4.

2. Mallon DP, Kingswood SC. Antelopes: North Africa, the Middle East, and Asia: lucn; 2001.

3. Habibi K. Mammals of Afghanistan: zoo outreach organisation with assistance from US fish and Wildlife Service; 2003.

4. Akbari H, Moradi HV, Sarhangzadeh J, Esfandabad BS. Population status, distribution, and conservation of the Chinkara, Gazella bennettii, in Iran (Mammalia: Bovidae). Zool Middle East. 2014; 60(3):189-94.

5. Dookia S, Rawat M, Jakher G, Dookia B. Status of the Indian Gazelle (Gazella bennettii Sykes, 1831) in the Thar desert of Rajasthan, India. Faunal Ecology and Conservation of the Great Indian Desert: Springer. 2009; 193-207.

6. Smith DH, Converse SJ, Gibson KW, Moehrenschlager A, Link WA, Olsen $\mathrm{GH}$ et al. Decision analysis for conservation breeding: maximizing production for reintroduction of whooping cranes. J Wildlife Manage. 2011; 75(3):501-8.

7. Bhowmik M. Disease spectrum and fawn mortality of hog deer (Axis porcinus) in Eastern Himalayan region. Tigerpaper. 2000; 27(4):17-20.

8. Meng $X$, Yang $H$, Yang $Q$, Feng $Z$, Peng $X$, Perkins GC. Preliminary findings of behavioral patterns in captive alpine musk deer (Moschus sifanicus) and prospects for future conservation. Turk J Vet Anim Sci. 2010; 34(2):111-7. 
9. Jakher G, Dookia S, Dookia B. Herd composition and population dynamics of Indian Gazelle (Gazella bennetti) (Sykes, 1831) in Gogelao Enclosure (Nagaur), Rajasthan. Zoos Print J. 2002; 17(11):9368.

10. Curio E. Conservation needs ethology. Trends Ecol Evol. 1996; 11(6):260-3.

11. Andersen IL, Nævdal E, Bøe KE, Bakken M. The significance of theories in behavioral ecology for solving problems in applied ethology-possibilities and limitations. Appl Anim Behav Sci. 2006; 97(1):85-104.

12. Håkansson J, Bratt $C$, Jensen $P$. Behavioural differences between two captive populations of red jungle fowl (Gallus gallus) with different genetic background, raised under identical conditions. Appl Anim Behav Sci. 2007; 102(1):24-38.

13. Price EO. Behavioral genetics and the process of animal domestication. 1999; 65(3):245-71.
14. Wallace M. Retaining natural behaviour in captivity for reintroduction programmes. Conservation Biology Series-Cambridge-2000; 300-14.

15. Zhang B. The taming and raising of musk deer. Agriculture, Press, Beijing. 1979.

16. Harri M, Mononen J, Ahola L, Plyusnina I, Rekilä T. Behavioural and physiological differences between silver foxes selected and not selected for domestic behaviour. Anim Welfare. 2003; 12(3):305-14

17. Parry-Jones R, Wu JY. Musk deer farming as a conservation tool in China: Traffic East Asia; 2001.

18. Clutton-Brock TH, Lukas D. The evolution of social philopatry and dispersal in female mammals. Mol Ecol. 2012; 21: 472-92.

19. Berger-Tal O, Polak T, Oron A, Lubin Y, Kotler B P, Saltz $D$. Integrating animal behavior and conservation biology: A conceptual framework. Behav Ecol. 2011; 22(2), 236-9. 